\title{
Improving Shelter Welfare with Online Training Focused on the Association of Shelter Veterinarians' Guidelines
}

\author{
Jennifer Lynn Federico ${ }^{1,}$, , Nicolette Petervary ${ }^{2}$, Ron Banks ${ }^{3}$ \\ ${ }^{1}$ Department of Environmental Services, Wake County, Raleigh, USA \\ ${ }^{2}$ National Institutes of Health, Office of Laboratory Animal Welfare, Bethesda, USA \\ ${ }^{3}$ Division of Comparative Medicine, Department of Pathology, The University of Oklahoma Health Sciences Center, Oklahoma, USA
}

Email address:

Jennifer.federico@wakegov.com (J. L. Federico)

${ }^{*}$ Corresponding author

\section{To cite this article:}

Jennifer Lynn Federico, Nicolette Petervary, Ron Banks. Improving Shelter Welfare with Online Training Focused on the Association of Shelter Veterinarians' Guidelines. Animal and Veterinary Sciences. Vol. 8, No. 1, 2020, pp. 1-13. doi: 10.11648/j.avs.20200801.11

Received: October 23, 2019; Accepted: January 3, 2020; Published: January 31, 2020

\begin{abstract}
Shelter care of animals and shelter medicine are both a growing field with expectations of improved welfare for shelter animals. The Association of Shelter Veterinarians (ASV) published The Guidelines for Standards of Care in Animal Shelters in 2010. The ASV Guidelines outline minimum care expectations for shelters, using a 'must,' 'should,' and 'ideal' ranking. Frequently shelters have limited resources, staff with a variety of training or experience, and often only consulting veterinarians - or even no veterinary coverage in certain locations. The ASV Guidelines are open access, freely available, and provide a roadmap for skills expectations. We performed comprehensive training searches to determine how available training could be crafted to meet or exceed the ASV Guidelines. The search for internet training resources was moderately successful. While useful resources were discovered, many others known to be available by the authors were not included in the search results. This article reviews our search methodology, reporting what was found and identifying certain 'missed' training activities. We then outline a basic online training program which could address each major topic identified in the ASV Guidelines and offer encouragement for shelter managers seeking to enhance local training experiences.
\end{abstract}

Keywords: Animal Shelter, Welfare, Online Training, Association of Shelter Veterinarians

\section{Introduction}

\subsection{Current State of Sheltering}

Animal shelters are typically understaffed and underfunded. The average operating cost in 2018 for North Carolina shelters was $\$ 664,166.95$, with an average intake of 2935 dogs and cats, based on data from eighty-two reporting shelters. On average, North Carolina shelters spent approximately \$226 per animal. Operating expenses in North Carolina ranged from $\$ 125$ (total intake of 11 dogs and cats) to $\$ 6,377,414$ (total intake of 9,811 dogs and cats) [1]. While detailed operational descriptions were not included in the NCDA report, clearly fiscal resources for basic sanitation, feed, and water would consume more than $\$ 11$ per animal, which is the calculated average cost per animal expense for the shelter with the $\$ 125$ operating expense. Turner, et al inferred that shelter staffing, time allocations, and skill set were also quite variable amongst organizations [2].

In 2010, the Association of Shelter Veterinarians (ASV) produced The Guidelines for Standards of Care in Animal Shelters. Animal sheltering does not have any official oversight to ensure appropriate treatment and advocate for the care and welfare of animals within animal shelters. The Guidelines came about to help organizations maintain high standards of animal welfare and adopt best practices. According to the Association of Shelter Veterinarians (ASV) Guidelines for Standards of Care in Animal Shelters [3]: "The goal of the ASV $<$ Guidelines $>$ was to provide information that will help any animal welfare entity meet the physical, mental and behavioral needs of the animals in their care. The Guidelines were developed to provide a tool that would allow communities and animal welfare organizations of all sizes, whether a large organization, a small home-based effort or something in between...". The Guidelines can be used by the shelter with eleven animals as well as the one taking in 9,811. 
In addition to the above, the Guidelines were also chosen to guide our training activities since the Guidelines are based on the Five Freedoms [4]. The Five Freedoms were originally prepared for agricultural species and have been accepted in non-agricultural settings since they are sufficiently broad enough that they may be applied to all animals in any setting. The application is appropriate since all animals have an interest in 1) Freedom from Hunger and Thirst, 2) Freedom from Discomfort, 3) Freedom from Pain, Injury or Disease, 4) Freedom to Express Normal Behavior, and 5) Freedom from Fear and Distress.

All shelters have a desire to assure good animal welfare by fulfilling the Five Freedoms, or a similarly declared code of care conduct. Unfortunately, shelters typically lack staff and funding to effectively provide for focused or enhanced animal care training for staff [5]. Returning to our North Carolina example, only 31 out of 82 , or $38 \%$ of the shelters had funding that exceeded the average cost per animal [1]. Since basic sanitation, feeding, and watering as priority consumes the majority of shelter budgets, training of staff and the resultant improvement of welfare which often accompanies improved animal welfare often becomes a secondary, or even tertiary concern.

\subsection{Online Search for Training}

In order to determine what online resources were available for development of shelter training programs, an online search was completed in September 2019. The search was done using "incognito" in Chrome. This process and methodology was chosen since most to all shelter managers would have access to the internet and internet searching tools, and one of our goals was to mirror what the shelter manager could find, in addition to what we might supplement. Incognito allows internet searching without tracking your current searches or using previous browser history, so one could search frequently and not be confined to prior searches or prior search reports. It is also beneficial since search engines are unable to gather prior search information which could alter or customize future search results. Main chapter titles in the ASV Guidelines were used as core search criteria, and each search also included "animal shelter" and "online training" as Boolean indicators. For example, a search performed included "animal shelter" AND "sanitation" AND "online training" to maximize the numbers of 'hits,' realizing such an approach could minimize specificity and report many 'false positives,' thereby requiring substantial effort to distill the valuable from the less useful. The results of each search were analyzed and 'hits' categorized. As would be expected, there was occasional overlap between topics.

Search results were reviewed in detail to investigate efficacy, accuracy, and applicability for shelter staff training. Ultimately, an example training program was developed that outlined applicable online content referenced to each section of the ASV Guidelines.
By documenting shelter training, either by use of this example program or focal program design, shelter leaders can identify trends or develop local staffing needs, identify validated training resources to address the trends or needs and ultimately enhance good animal welfare and care provisions while assuring the ASV Guidelines are met.

\section{Materials \& Methods}

\subsection{Search Related to ASV Guidelines}

Using publicly available search tools, we completed a search for online resources related to the chapters in the ASV Guidelines. Sites were evaluated based on free (or low cost), availability and accessibility from an internet connection, and content quality. Reliability of the website was considered and included the following sources: universities; governmental organizations; professional groups; and commonly-recognized non-profit organizations involved with animal sheltering. As described above, each chapter title from the ASV Guidelines were used as part of the search criteria in addition to the words, "animal shelter" and "online training". Chapter titles with two focal points, such as Management and Record Keeping, were divided into two searches, one with management and another using record keeping. This method was employed to gather maximal site information related to the items covered within the chapter.

Table 1. Chapter Titles from ASV Guidelines.

\begin{tabular}{l}
\hline Chapter Titles \\
\hline Management and Record Keeping \\
Facility Design and Environment \\
Population Management \\
Sanitation \\
Medical Health and Physical Well-being \\
Behavioural Health and Mental Well-being \\
Group Housing \\
Animal Handling \\
Euthanasia \\
Spaying and Neutering \\
Animal Transport \\
Public Health
\end{tabular}

The initial search was limited to the first three pages returned as those 'hits' were most relevant. A total of 487 resources were returned. Each resource was evaluated as 'relevant' or 'not relevant' to the topic searched and whether it appeared a plausible resource for shelter staff training. Table 2 outlines the initial measures used to evaluate the sites individually.

\subsection{Evaluation and Review of Relevant Sites}

Eighty-six resources were considered relevant to the main search topic. Duplicates were then removed. The twenty-eight relevant sites were then further evaluated for content, cost and reliability. 
Table 2. Criteria used to determine 'Non-Relevant' and 'Relevant' Content.

\begin{tabular}{ll}
\hline Non-Relevant & Relevant \\
\hline 1. Any website with the word "Ad" before it & University sites \\
2. Content not related to animal sheltering & 2. Governmental organization \\
3. Content only relevant to specific government agency producing the site & 3. Professional groups \\
4. Personal views & 4. Well-known non-profit groups involved with animal shelters \\
5. Career Tests & 5. Conference related to animal sheltering \\
6. Career Search Sites & 6. Degree / Certificate seeking programs related to topics \\
7. Job advertisements & 7. Any in-person training related to the topic no matter the cost \\
8. Shelter software & \\
9. Articles with only a mention of the topic, but lacking specific information related to the topic & \\
10. Individual shelter manuals related to programs outside the scope of the ASV & \\
Guidelines (i.e. volunteer, animal control, etc.) & \\
11. Disaster Preparedness / sheltering of animals in a disaster & \\
12. Social media site (i.e. Facebook, Pinterest, etc.) & \\
13. Newsletters / Annual Reports & \\
\hline
\end{tabular}

Of the 28 relevant sites, ten were linked to paid courses or conferences. Some of this training was available online, however, the majority required travel. The courses and conferences returned are outlined, along with their website, in
Table 3. Due to the cost, these courses were not included in the training program outlined as the goal of this investigation was for a no cost or minimal cost training option.

Table 3. Paid Courses and Conferences.

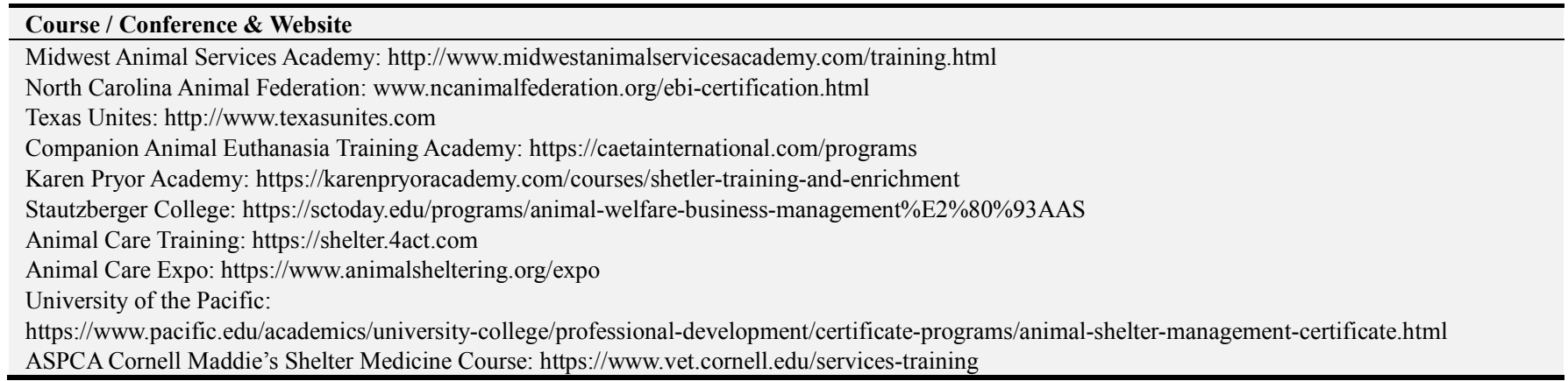

Of the 28 relevant websites, the relevancy to each main topic was reviewed. The sites were closely reviewed to determine if their content would add to the training program being developed and how many topics were addressed by that site. Some sites were found under one search but noted to cover topics in another search criteria. The websites that related to conferences, which have changing topics annually, were listed as "maybe," since the applicability of a changing conference, especially for future not reported conferences, was impossible to evaluate. The results are shown in Figure 1.

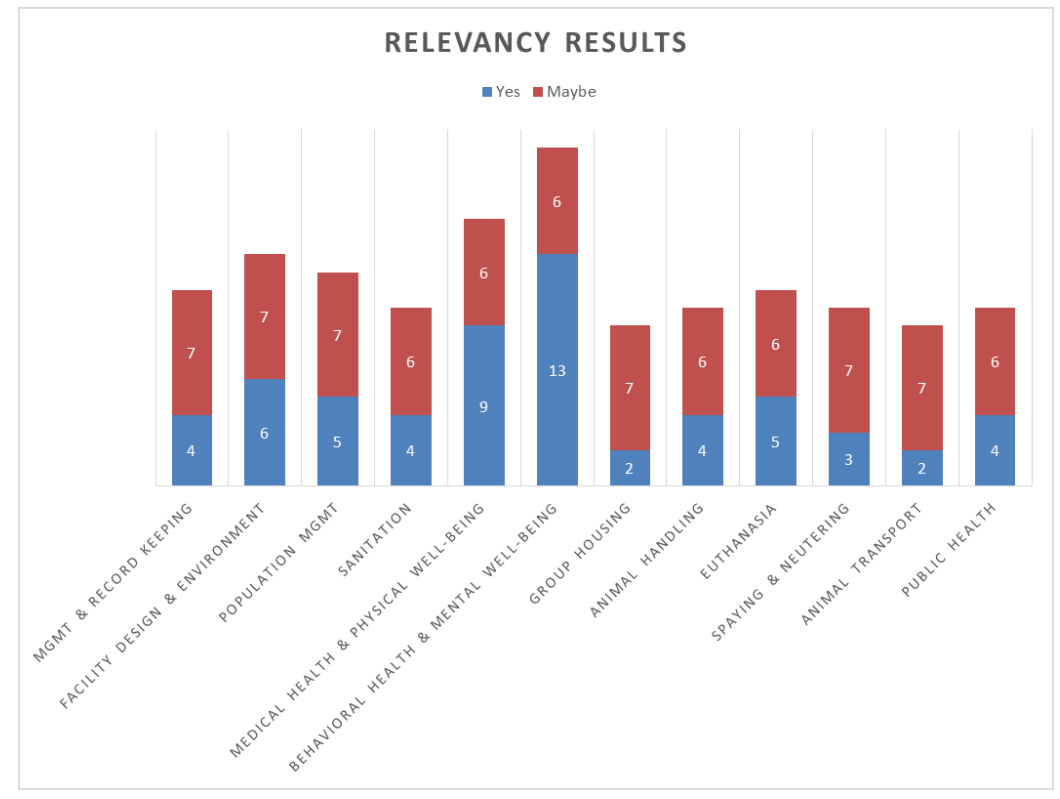

Figure 1. Relevancy of websites to ASV Guidelines Topic. 
It was not surprising that the most common resources pertained to medical and behavioral health. Medical health and behavioral health is where the greatest needs exist for most shelter staff, since many shelters do not have veterinarians on staff and frequently rely upon non-veterinary staff for frontline or cage-side assessment.

\section{Results}

\subsection{Resources Not Found in Search}

Although many sites were found, there were limitations to the analysis. The returned search results did not include prominent resources known to the authors. One example includes specific webinars on sites that were found but did not guide a user to this resource. Some websites were completely missed and included the Centers for Disease Control (CDC) regarding public health, the Drug Enforcement Agency (DEA) regarding record keeping, American Humane Association for multiple topics and Dogs Playing for Life for behavioral health. This suggests that shelters may miss training opportunities or critical information, if searches are not performed by experienced or field knowledgeable shelter staff. The additional resources were included in the summation of all training opportunities at the end of this report. The training program outlined later in this report only includes sources that meet the requirements for reliability and content relevance, as previously described.

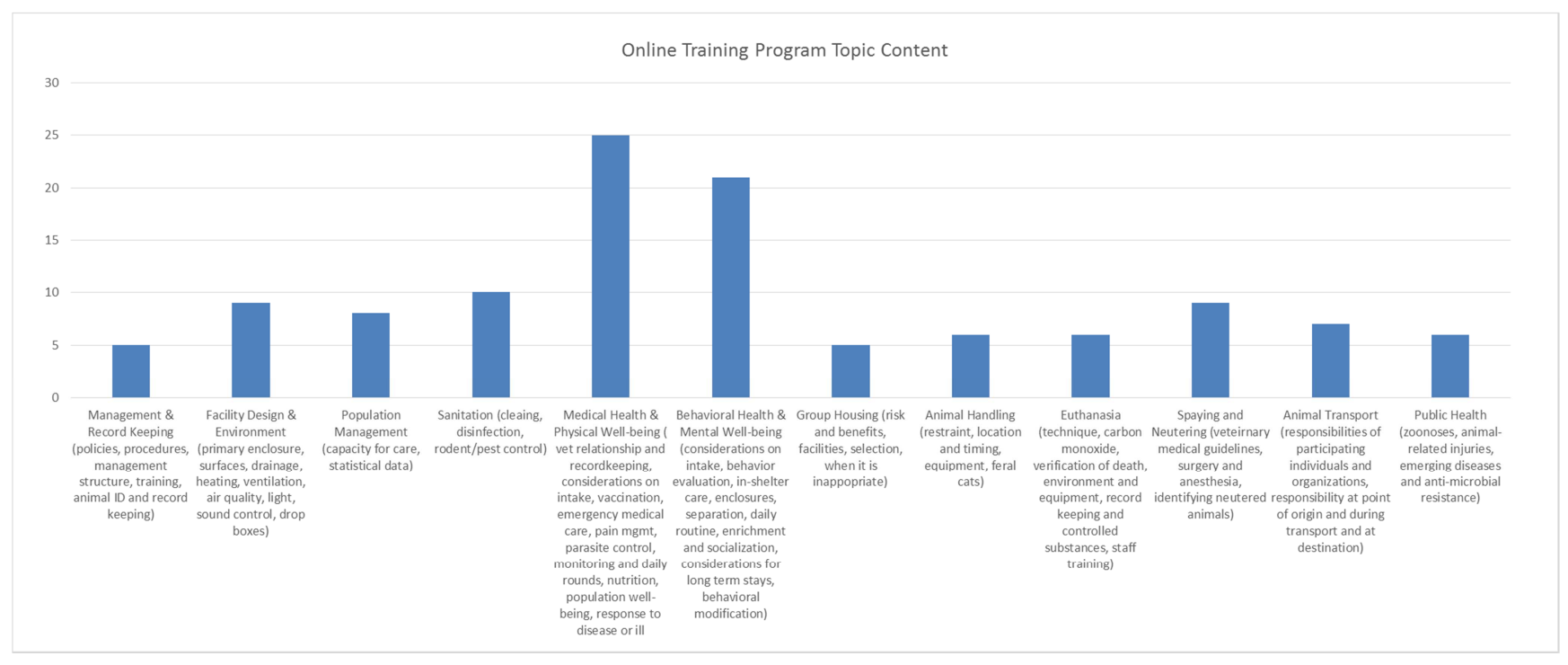

Figure 2. Content for Training Program.

\subsection{Euthanasia Training}

Large numbers of animals are regularly euthanized in shelters. In our focal example, annually approximately $30 \%$ of the animals entering North Carolina shelters (82 NC shelters reported their numbers) are euthanized. While responsibility for euthanasia may vary state to state regarding who may be authorized to perform animal euthanasia, euthanasia has many technical, psychological, and societal implications (compassion fatigue) for which most non-veterinary staff are poorly prepared. Euthanasia training may be one area where more online training would be highly beneficial for shelter staff. While certain training was identified, we also noted that the specific training was not generally identified in the topic of a title - ASPCAPro's Webinar entitled "Guidelines for Standards of Care in Animal Shelters". Nowhere does this title include euthanasia, although the webinar includes approximately thirty minutes on euthanasia techniques which are critical to shelters that perform euthanasia.

Open admission shelters, according to the Association of Shelter Veterinarians are "traditionally operated by municipalities or hold animal control contracts for municipalities" [6]. These shelters typically accept owner surrenders, strays and abandoned animals - regardless of adoptability, health or temperament. The importance of understanding and performing quality euthanasia is imperative, and especially in the absence of veterinarians. The criticality of having access to good online training becomes even more substantial in these circumstances.

\subsection{Limitations of Findings}

There was a relative paucity of quality resources on zoonotic risks in shelters, especially considering the importance of this topic for staff and potential adopters. Rabies information is critical for all shelters. Being able to recognize the symptoms, understanding vectors (or fomites in some circumstances) and having the knowledge for determining how to handle exposures or how exposures may affect placement is critical to shelter staff. The Compendium of Animal Rabies Prevention and Control, 2016, produced by the National Association of State Public Health Veterinarians, has sections specifically regarding stray animals and acknowledge that stray and feral cats pose a rabies exposure risk and advocate for vaccination [7].

We also note our focus was to investigate availability of 
shelter training, but our focus was further refined to the topics outlined in the ASV Guidelines. Even so, it is worth noting that the Guidelines do not include volunteer programs, foster programs, rescue program or animal control programs. Some shelters have all or some of the mentioned programs, and do need trained and knowledgeable personnel to staff those programs. As such, future research into quality training for these programs could be another review, which when supplemented by a proposed training program, could be another important resource for shelter staff.

\section{Discussion}

\subsection{Training Program}

Online training is enhancing other fields such as safety in the workplace, public health preparedness and response and prescribing competency in UK junior doctors. [8-10]. Public Health is seeing some of the same challenges as animal shelters, including limited funds and a small workforce [10]. The authors devised a training program (Appendix A) which could be engaged in the shelter community, regardless of size or scope, utilizing only free, open access content, and based on the topic categories in Figure 2. Shelters could assemble most training documents in binders of articles to read and webinars to view. For each of the recommended sites, additional areas of interest may be explored (e.g., fostering, volunteering, kitten specific information, etc.). The training proposal includes training in population management or identification of infectious disease - topics commonly limited for many shelter employees, especially if volunteers are utilized [2]. Free, online training may provide essential training for workers, while leveraging fiscal resources.

Appendix A outlines training material under each ASV category. Shelter leaders can choose which resources would be most effective for their shelter staff. Training plans may be scripted to specific shelter needs. For example, one shelter may have challenges with staff recognition of common shelter illnesses and designs a training program focused to address this shortcoming. In another shelter, concerns with animal handling skills may provide direction to craft a web training program specifically for this issue. All shelters may choose to develop a global training activity required of all new hires prior to direct contact with animals (an example program for new staff is listed in Table 4).

The 'new hire' training was ordered by significance for specified positions (not everyone needs to be trained on everything) and in the order that they should be completed. Shelter managers would need to accomplish a pre-work skills inventory to determine which modules or training tools would be most effective for the individual.

\subsection{Successful Online Training Programs}

The U.S. Department of Education has reported that students in online learning conditions performed just as well, and sometimes better, then students learning in a typical face-to-face classroom setting [11]. Developing an online training program is the first step in augmenting the knowledge of your team. The next step is providing the team members with the right circumstances and time in order to complete the program. Ideally, time would be allocated for a team member to complete the training without interruptions and prior to a time when that skill or knowledge item would be required. One way to lay out the training is dividing the topics so that desired concepts can be learned in the time allotted. Shelter 'busy-ness' or training interruptions may cause an employee to discontinue the training, fail the complete the training, or extend the time required to accomplish the training [12]. Collectively this results in unsuccessful training, frustrated students, and failed work performance, none of which lead to a positive learning experience or effectively performing team member.

The organization's culture and excitement for employee development may also impact a team member's perspective on training. Online training is appealing due to the self-paced format [13]. However, some team members will excel if given in-person time to discuss what they have learned. One option is to have a small subset of team members perform the same training module and then, after completing the training, meet with their supervisor to review questions regarding the training and summarize the major topics of the training. This approach can reinforce the training objectives, substantiate the institutional commitment to developing skilled staff, help solidify what they have learned. Students who recognize that the senior team members are invested in their learning will become more dedicated employees.

Training assignments should not be completed in one setting, or even over a short series of days. Giving team members time for absorbing the recently learned information helps it become retainable knowledge. Generally, it is best to allow team members short periods of training separated by breaks over hours or days [14]. An entire training program generally should not be completed in one sitting.

\subsection{Local Resources}

Individual shelters may augment training by inviting local experts to provide hands-on training. This would be especially critical to euthanasia techniques and animal handling. Studies have found that a melding of online training along with traditional learning environments may produce the best outcomes [15]. Most communities have access to veterinarians and professional dog trainers which, in addition to providing hands on training, provide community partnership and an active interest in the well-being of the animals within the shelter. Another option is to partner with national entities, such as the American Society for the Prevention of Cruelty to Animals (ASPCA) or Humane Society of the United States (HSUS), to provide local training in specific topics open to many local shelters. These trainings are typically provided free of charge to attendees, especially if a need is demonstrated. 
Table 4. Example Training Program for New Staff (See Appendix B for outlined training related to this figure).

\begin{tabular}{lll}
\hline Animal Care Attendants & Technicians (performing intake, euthanasia and veterinary care) & Management Team \\
\hline Sanitation & Animal Handling & Management \& Record Keeping \\
Animal Handling & Medical Health \& Physical Well-being & Facility Design \& Environment \\
Medical Health \& Physical Well-being & Sanitation & Population Management \\
Behavioural Health \& Mental Well-being & Euthanasia & Sanitation \\
Group Housing & Spay \& Neuter (if performed at shelter) & Public Health \\
Public Health & Behavioural Health \& Mental Well-being & Medical Health \& Physical Well-being \\
Management \& Record Keeping & Population Management & Behavioural Health \& Mental \\
(specifically animal ID and record keeping) & Well-being & Euthanasia \\
& Public Health & Group Housing \\
& Management \& Record Keeping & Spay/Neuter (if performed at shelter) \\
& & Animal Transport (if performed) \\
\hline
\end{tabular}

In addition to assisting shelters with achieving ASV Guidelines, training programs as described above would empower team members to grow in their profession, along with possible upward mobility within the organization.

\section{Conclusion}

The challenges of finding web based free training opportunities are substantial. Even searches performed by seasoned professionals may not be complete regarding good training tools. Most shelter management or staff do not have the skill-set of identifying subtle or poorly advertised training opportunities available online.

The materials outlined are produced by experts in the field of sheltering are of no cost and are open access. These curated resources are provided with the goal of improving the care and welfare of shelter animals, especially for organizations with limited resources. Even so, due to continuous changes in internet content, searches should be updated periodically, content should be professionally reviewed regularly, and training programs using online materials should be updated accordingly.

While opportunities for free, open access shelter training online remain available, there is a scarcity of information in certain areas. More online content concerning topics such as euthanasia, animal handling, group housing, management and record keeping as well as public health remains a need for the shelter community.

The ASV Guidelines for Standards of Care in Animal Shelters serves as a valid and reliable framework for shelters wishing to build a training program. Quality education is essential for shelter staff to improve animal welfare and well-being [2] and can be provided inexpensively and effectively.

\section{Disclaimer}

Dr. Petervary contributed to this article in her personal capacity. The views expressed are her own and do not necessarily represent the views of the National Institutes of Health or the United States Government.

\section{Appendix}

\section{Appendix A: Training Program Following the ASV Guidelines Topics}

\section{Appendix A1: Management \& Record Keeping}

Table 5. Training Material Available for Management \& Record Keeping.

\begin{tabular}{l}
\hline Topics include: polices, procedures, management structure, training, animal ID and record keeping \\
\hline https://www.americanhumane.org/app/uploads/2016/08/op-guide-recordkeeping.pdf \\
https://www.animalsasia.org/cn/assets/pdf/1\%20Basic\%20Management\%20Guidelines\%20for\%20Dog\%20and\%20Cat\%20Shelters.pdf \\
https://www.aspcapro.org/webinar/20110224/shelter-guidelines-management-recordkeeping \\
https://www.aspcapro.org/webinar/20110331/shelter-guidelines-five-freedoms-and-shelter-wellness \\
https://www.americanhumane.org/publication/animal-shelter-operational-guide-record-keeping/
\end{tabular}

\section{Appendix A2: Facility Design \& Environment}

Table 6. Training Material Available for Facility Design \& Environment.

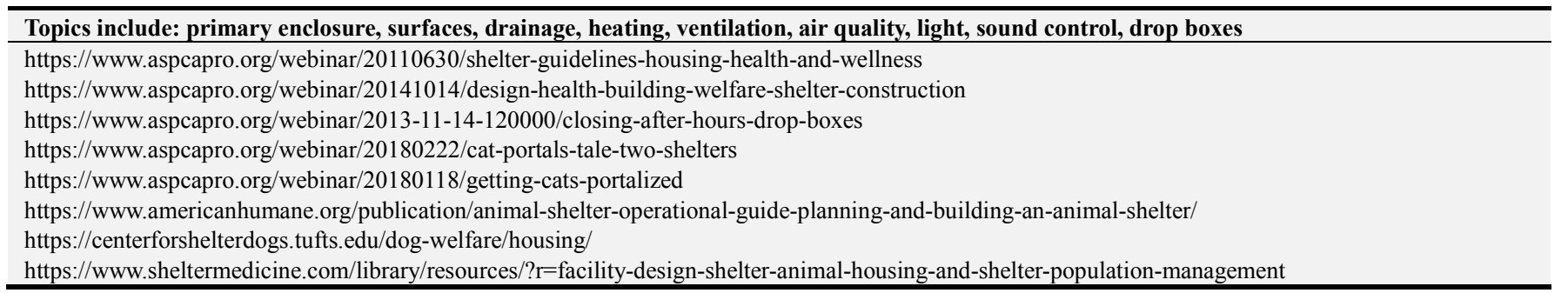




\section{Appendix A3: Population Management}

Table 7. Training Material Available for Population Management.

\begin{tabular}{l}
\hline Topics include: capacity for care, statistical data \\
\hline https://www.aspcapro.org/webinar/20160120/turning-data-lives-saved \\
https://www.aspcapro.org/webinar/20131120/calculating-your-humane-capacity \\
https://centerforshelterdogs.tufts.edu/dog-welfare/population-management/ \\
https://www.maddiesuniversity.org/Catalog/LrnrTab2332/catalog/LrnrCtrl2332/catalog/LrnrKC2332/true/FID2332/c4033591-0259-4flf-8ca9-2c896e940297 \\
https://www.maddiesuniversity.org/Catalog/LrnrTab2332/catalog/LrnrCtrl2332/catalog/LrnrKC2332/true/FID2332/0a8e2930-3b66-428b-a3f3-0dd60b2a2873 \\
https://www.sheltermedicine.com/library/resources/?r=overview-of-capacity-for-care-c4c \\
https://www.sheltermedicine.com/library/resources/?r=calculating-shelter-capacity \\
https://shelteranimalscount.org/
\end{tabular}

\section{Appendix A4: Sanitation}

Table 8. Training Material Available for Sanitation.

\begin{tabular}{l}
\hline Topics include: cleaning, disinfection, rodent/ pest control \\
\hline https://www.aspcapro.org/webinar/20110526/shelter-guidelines-sanitation \\
https://www.aspcapro.org/resource/shelter-health-disease-management/shelter-disinfectant-quick-reference \\
https://www.aspcapro.org/webinar/20170718/shelter-sanitation-2 \\
https://www.aspcapro.org/webinar/20170711/shelter-sanitation-1 \\
https://www.aspcapro.org/resource/sanitation-communal-cat-rooms \\
https://www.americanhumane.org/publication/animal-shelter-operational-guide-sanitation-and-disease-control-in-the-shelter-environment/ \\
https://sheltermedicine.vetmed.ufl.edu/files/2011/11/Cleaning_and_Disinfection_101.pdf \\
https://sheltermedicine.vetmed.ufl.edu/files/2011/10/Guidelines-for-Using-Bleach-updated.pdf \\
https://www.maddiesuniversity.org/Catalog/LrnrTab2332/catalog/LrnrCtrl2332/catalog/LrnrKC2332/true/FID2332/fe8f9d82-eb11-4ac8-adf9-239bc549f16c \\
https://www.sheltermedicine.com/library/resources/?r=sanitation-in-animal-shelters \\
\hline
\end{tabular}

\section{Appendix A5: Medical Health \& Physical Well-being}

Table 9. Training Material Available for Medical Health \& Physical Well-being.

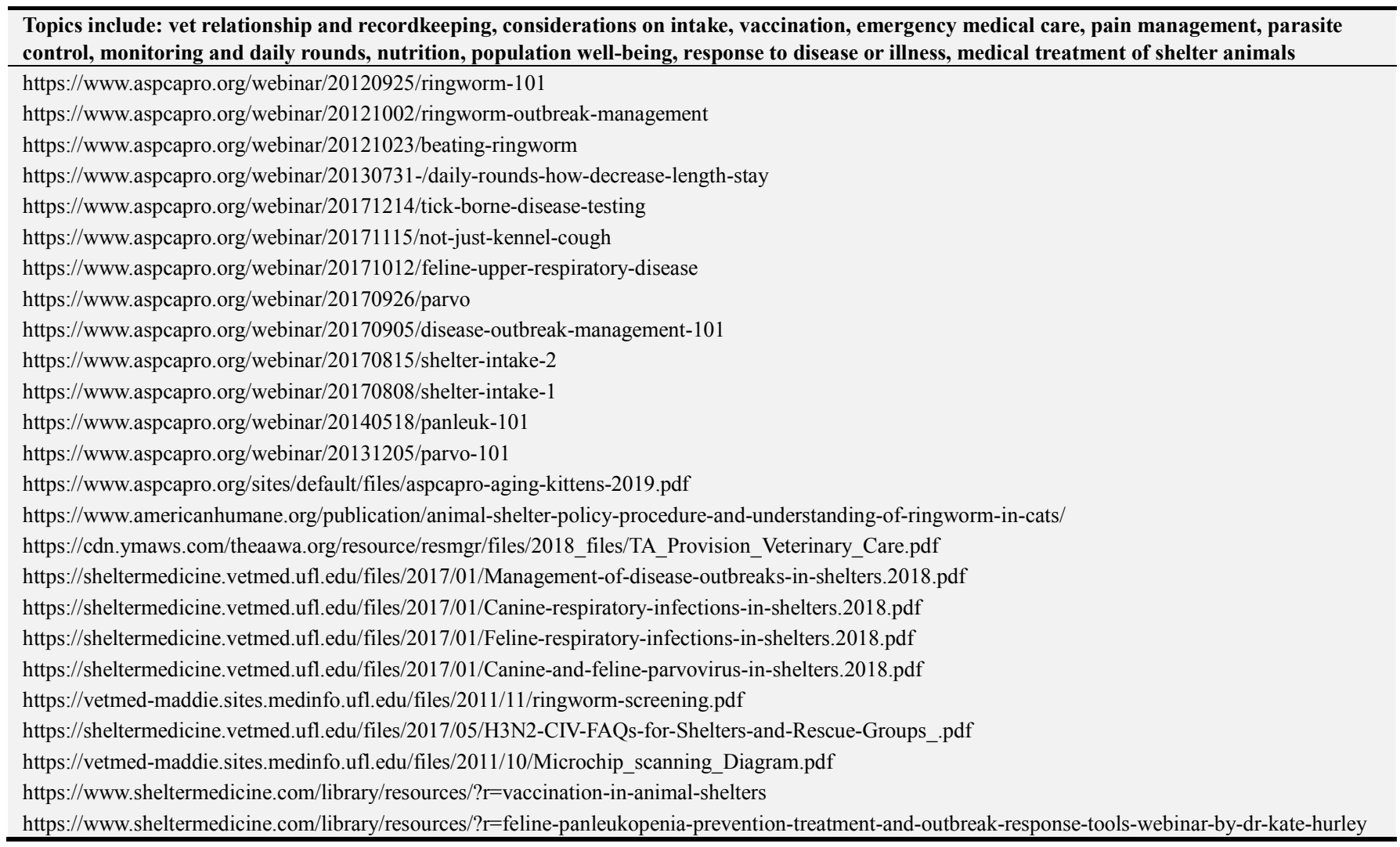




\section{Appendix A6: Behavioral Health \& Mental Well-being}

Table 10. Training Material Available for Behavioral Health \& Mental Well-being.

\begin{tabular}{l} 
Topics include: considerations on intake, behavior evaluation, in-shelter care, enclosures, separation, daily routine, enrichment and socialization, \\
considerations for long term stays, behavioral modification \\
\hline https://karenpryoracademy.com/courses/shelter-training-and-enrichment/ \\
https://www.aspcapro.org/webinar/20110728/shelter-guidelines-behavioral-health-shelter \\
https://cdn.ymaws.com/theaawa.org/resource/resmgr/files/2018_files/The_Association_Animal_BP.pdf \\
https://www.aspcapro.org/webinar/20180110/animal-enrichment-best-practices \\
https://www.aspcapro.org/webinar/20180124/plan-track-best-enrichment-program-ever \\
https://www.aspcapro.org/webinar/20180207/8-components-animal-enrichment-program \\
https://www.aspcapro.org/webinar/20180221/cheap-fun-enrichment-ideas \\
https://www.aspcapro.org/webinar/2018-11-13-120000/assessing-canine-behavior-shelter-how-collect-objective-info \\
https://www.aspcapro.org/webinar/2018-11-06-120000/assessing-canine-behavior-shelter-input-multiple-sources \\
https://www.aspcapro.org/webinar/20150909/animal-behavior-shelters \\
https://www.aspcapro.org/webinar/20130912/canine-behavior-and-acoustics \\
https://www.aspcapro.org/webinar/20130919/is-cat-feral \\
https://www.aspcapro.org/webinar/20130618/stress-reduction-enrichment-shelter-cats \\
https://www.aspcapro.org/webinar/20130129/enrichment-shelter-dogs \\
https://www.americanhumane.org/publication/animal-shelter-operation-guide-behavior-assessment-programs/ \\
https://sheltermedicine.vetmed.ufl.edu/shelter-services/tools-tips-fact-sheets/shelter-enrichment-resources/ \\
https://centerforshelterdogs.tufts.edu/dog-behavior/dog-communication-and-body-language/ \\
https://www.maddiesuniversity.org/Catalog/LrnrTab2332/catalog/LrnrCtrl2332/catalog/LrnrKC2332/true/FID2332/7a1c3803-13a3-45c8-b81a-1cf2c4376de9 \\
https://www.maddiesuniversity.org/Catalog/LrnrTab2332/catalog/LrnrCtrl2332/catalog/LrnrKC2332/true/FID2332/34902747-16e4-4a56-a620-a5a28aab7724 \\
https://www.maddiesuniversity.org/Catalog/LrnrTab2332/catalog/LrnrCtrl2332/catalog/LrnrKC2332/true/FID2332/f929a9b1-3d7f-4fb9-8679-b5494d839eb8 \\
https://dogsplayingforlife.com/dpfl-manual/
\end{tabular}

\section{Appendix A7: Group Housing}

Table 11. Training Material Available for Group Housing.

\begin{tabular}{l}
\hline Topics include: risk and benefits, facilities, selection, when it is inappropriate \\
\hline https://www.aspcapro.org/webinar/20111215/shelter-guidelines-group-housing \\
https://centerforshelterdogs.tufts.edu/blog/group-housing-part-one/ \\
https://www.petfinder.com/pro/for-shelters/guidelines-communal-housing/ \\
https://www.petfinder.com/pro/for-shelters/colony-housing-shelter-cats/ \\
\hline
\end{tabular}

\section{Appendix A8: Animal Handling}

Table 12. Training Material Available for Animal Handling.

\begin{tabular}{l}
\hline Topics include: restraint, location and timing, equipment, feral cats \\
https://www.aspcapro.org/webinar/20110929/shelter-guidelines-animal-handling \\
https://www.aspcapro.org/webinar/20120418/canine-body-language \\
https://www.aspcapro.org/webinar/20120426/human-body-language-and-dog-behavior \\
https://www.aspcapro.org/webinar/20120510/defensive-dog-handling \\
https://www.aspcapro.org/webinar/20130409/humane-feline-handling-101 \\
https://www.aspcapro.org/webinar/20130402/feline-communication \\
\hline
\end{tabular}

\section{Appendix A9: Euthanasia}

Table 13. Training Material Available for Euthanasia.

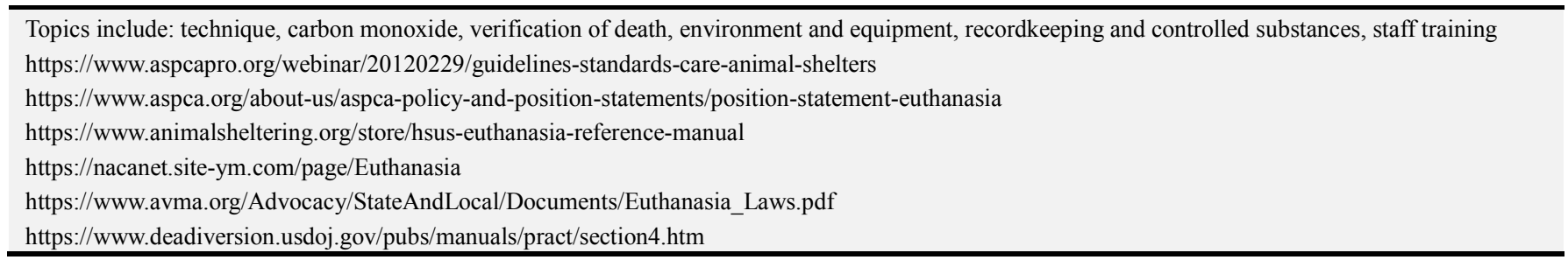




\section{Appendix A10: Spaying and Neutering}

Table 14. Training Material Available for Spaying and Neutering.

\begin{tabular}{l}
\hline Topics include: veterinary medical guidelines, surgery and anesthesia, identifying neutered animals \\
\hline https://www.aspcapro.org/webinar/20121117/shelter-guidelines-spayneuter \\
https://www.aspcapro.org/webinar/20180227/feline-fix-five \\
https://www.aspcapro.org/webinar/20160309/ergonomics-surgeons \\
https://www.aspcapro.org/webinar/20151116/spayneuter-clinic-anesthetic-protocols \\
https://www.aspcapro.org/resource/spayneuter-clinic-drug-charts-logs \\
https://www.aspcapro.org/webinar/20121117/shelter-guidelines-spayneuter \\
https://www.aspcapro.org/training-online-courses/spayneuter-online-courses \\
https://avmajournals.avma.org/doi/pdf/10.2460/javma.249.2.165 \\
https://www.aspcapro.org/training-site-training-spayneuter-clinic-mentorship/spayneuter-mentorship-materials \\
\hline
\end{tabular}

\section{Appendix A11: Animal Transport}

Table 15. Training Material Available for Animal Transport.

\begin{tabular}{l} 
Topics include: responsibilities of participating individuals and organizations, responsibility at point of origin and during transport and at \\
destination \\
\hline https://www.aspcapro.org/webinar/20110825/shelter-guidelines-animal-transport \\
https://www.aspcapro.org/webinar/20161207/introduction-transport-best-practices-1 \\
https://www.aspcapro.org/webinar/20170104/transport-agency-and-community-2 \\
https://www.aspcapro.org/webinar/20170125/building-relationships-transport-programs-3 \\
https://www.aspcapro.org/webinar/20170215/bon-voyage-transported-animals-4 \\
https://www.aspcapro.org/webinar/20170308/transport-vaccinations-funding-goals-5 \\
https://www.aspcapro.org/webinar/20170329/how-transport-cats-6
\end{tabular}

\section{Appendix A12: Public Health}

Table 16. Training Material Available for Public Health.

\begin{tabular}{l} 
Topics include: zoonoses, animal-related injuries, emerging diseases and antimicrobial resistance \\
https://www.aspcapro.org/webinar/20111013shelter-guidelines-healthy-and-safe-environment \\
https://www.americanhumane.org/publication/animal-shelter-operation-guide-companion-animal-zoonotic-diseases/ \\
https://www.sheltermedicine.com/library/resources/?r=zoonotic-disease-prevention-in-shelters \\
https://blogs.cdc.gov/publichealthmatters/2018/05/national-pet-week/ \\
https://www.cdc.gov/healthypets/health-benefits/index.html \\
https://www.cdc.gov/healthypets/specific-groups/veterinarians.html \\
\hline
\end{tabular}

\section{Appendix A13: Primary Resources for Shelter Staff (in no Particular Order)}

Table 17. Primary Resources for Shelter Staff (in no particular order).

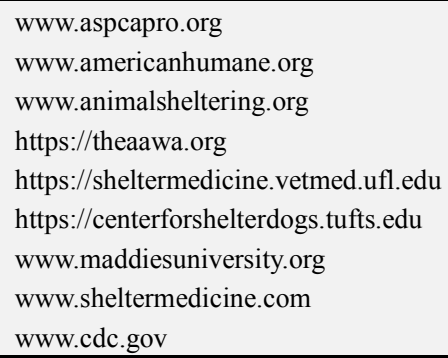

\section{Appendix A14: Paid Certificate / Masters Programs}

Table 18. Paid Certificate / Masters Programs Available.

\begin{tabular}{l}
\hline Animal Shelter Management Certificate (Online) \\
\hline https://www.pacific.edu/academics/schools-and-colleges/benerd-college/professional-development/certificate-programs/animal-shelter-management-certificate.html \\
Animal Welfare Business Management - AAS (Online) https://sctoday.edu/programs/animal-welfare-business-management\%E2\%80\%93AAS \\
Maddie's Professional Certificate in Shelter Medicine (Online) https://sheltermedicine.vetmed.ufl.edu/education/on-campus-certificate/ \\
\hline
\end{tabular}




\section{Appendix B: Training Outlines per the Training Program Outlined in Appendix A}

\section{Appendix B1: Training Outline for Animal Care Attendants}

Table 19. Example of a Training Outline for Animal Care Attendants.

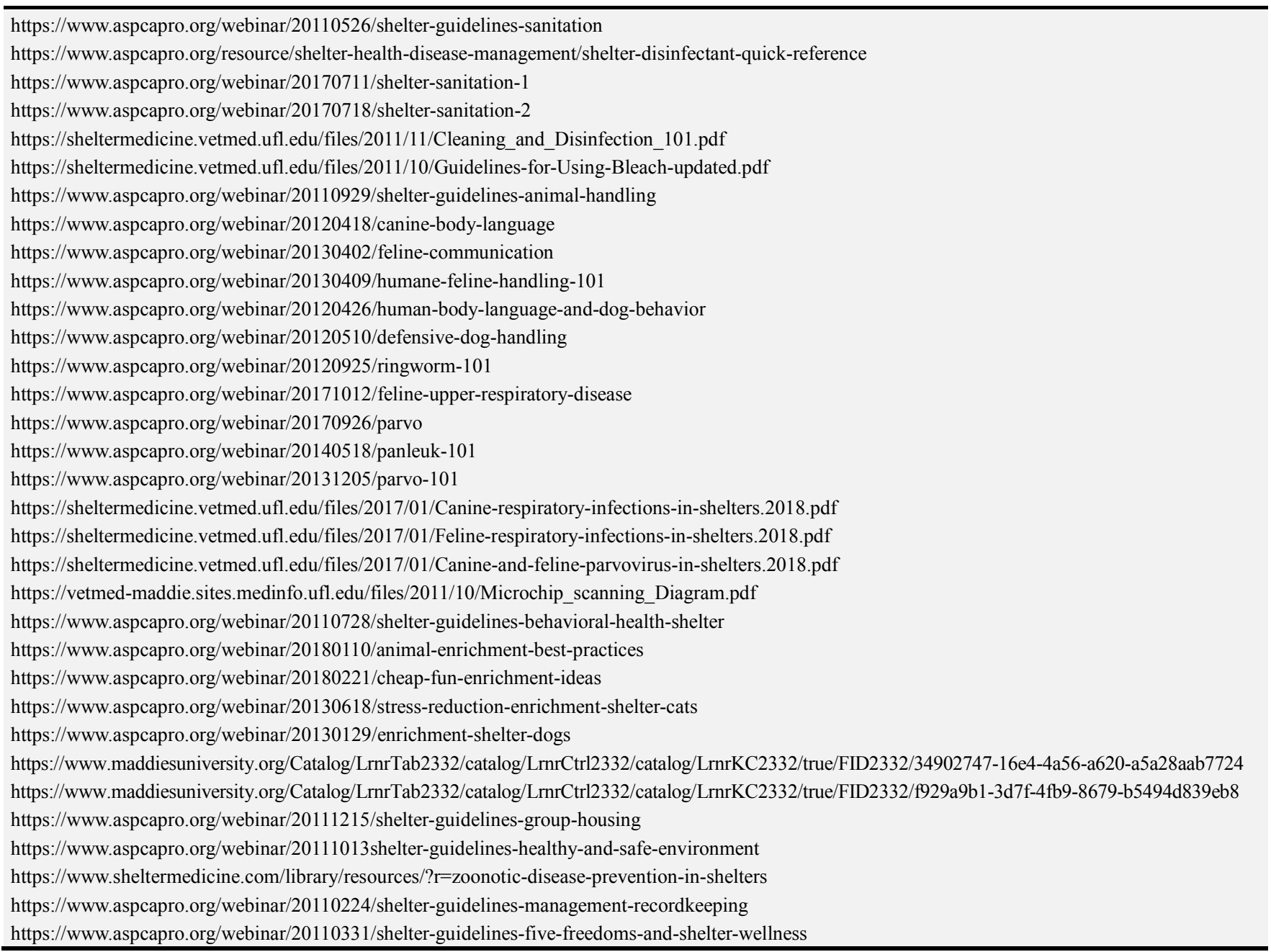

\section{Appendix B2: Training Outline for Technicians}

Table 20. Example of a Training Outline for Technicians.

\footnotetext{
https://www.aspcapro.org/webinar/20110929/shelter-guidelines-animal-handling

https:/www.aspcapro.org/webinar/20120418/canine-body-language

https:/www.aspcapro.org/webinar/20120426/human-body-language-and-dog-behavior

https:/www.aspcapro.org/webinar/20120510/defensive-dog-handling

https://www.aspcapro.org/webinar/20130409/humane-feline-handling-101

https://www.aspcapro.org/webinar/20130402/feline-communication

https:/vetmed-maddie.sites.medinfo.ufl.edu/files/2011/10/Microchip_scanning_Diagram.pdf

https:/www.sheltermedicine.com/library/resources/?r=vaccination-in-animal-shelters

https://www.aspcapro.org/webinar/20120925/ringworm-101

https://www.aspcapro.org/webinar/20121002/ringworm-outbreak-management

https:/www.aspcapro.org/webinar/20130731-/daily-rounds-how-decrease-length-stay

https://www.aspcapro.org/webinar/20171115/not-just-kennel-cough

https:/www.aspcapro.org/webinar/20171012/feline-upper-respiratory-disease

https://www.aspcapro.org/webinar/20170926/parvo

https://www.aspcapro.org/webinar/20170905/disease-outbreak-management-101

https://www.aspcapro.org/webinar/20170815/shelter-intake-2

https://www.aspcapro.org/webinar/20170808/shelter-intake-1

https://www.aspcapro.org/webinar/20140518/panleuk-101
} 


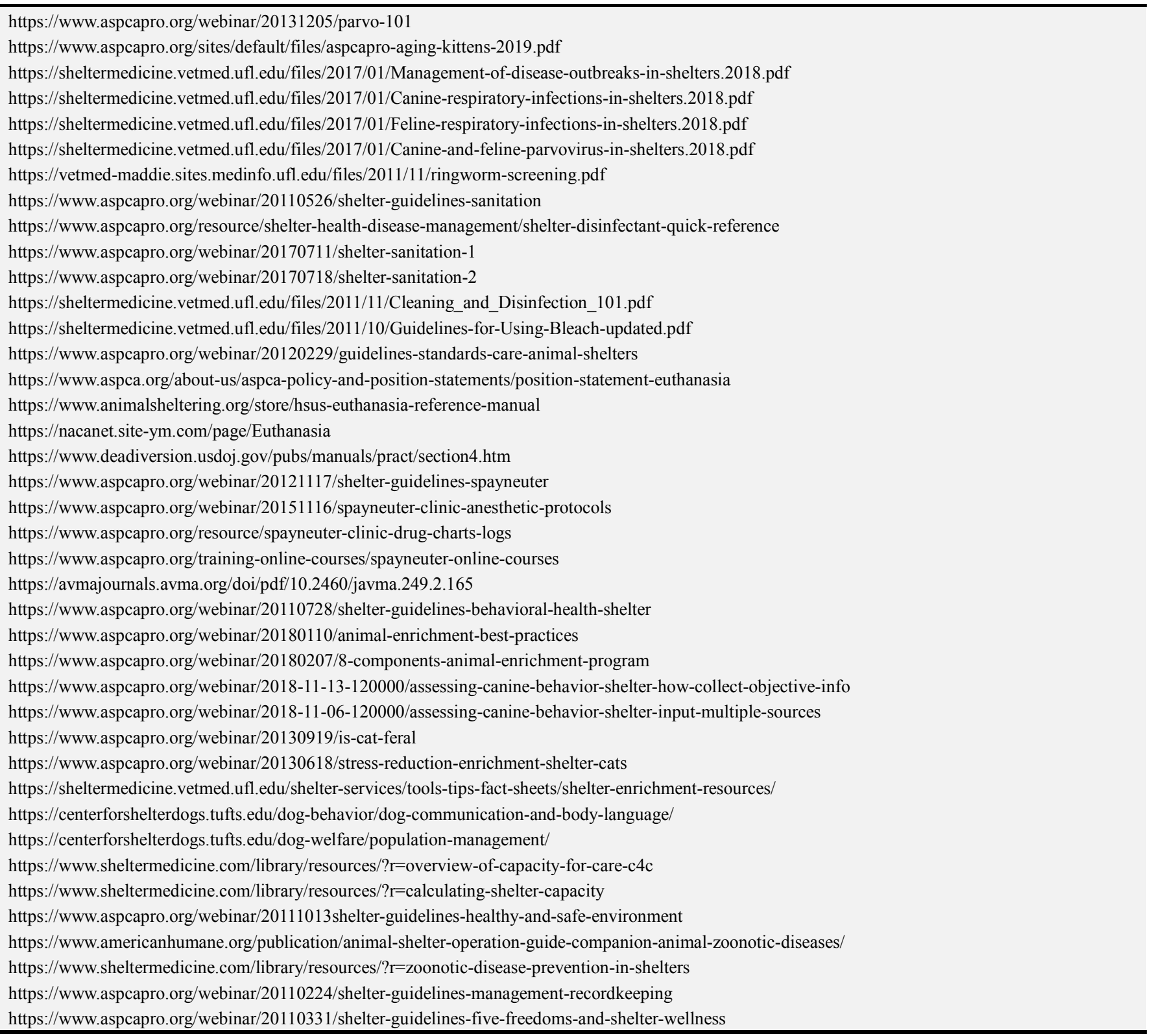

\section{Appendix B3: Training Outline for Management}

Table 21. Example of a Training Outline for Management.

\footnotetext{
https://www.aspcapro.org/webinar/20110224/shelter-guidelines-management-recordkeeping https://www.aspcapro.org/webinar/20110331/shelter-guidelines-five-freedoms-and-shelter-wellness https://www.americanhumane.org/publication/animal-shelter-operational-guide-record-keeping/ https://www.aspcapro.org/webinar/20110630/shelter-guidelines-housing-health-and-wellness https://www.aspcapro.org/webinar/20141014/design-health-building-welfare-shelter-construction https://www.aspcapro.org/webinar/20180222/cat-portals-tale-two-shelters https://www.americanhumane.org/publication/animal-shelter-operational-guide-planning-and-building-an-animal-shelter/ https://centerforshelterdogs.tufts.edu/dog-welfare/housing/ https://www.sheltermedicine.com/library/resources/?r=facility-design-shelter-animal-housing-and-shelter-population-management https://www.aspcapro.org/webinar/20160120/turning-data-lives-saved https://www.aspcapro.org/webinar/20131120/calculating-your-humane-capacity https://centerforshelterdogs.tufts.edu/dog-welfare/population-management/ https://www.sheltermedicine.com/library/resources/?r=overview-of-capacity-for-care-c4c https://www.sheltermedicine.com/library/resources/?r=calculating-shelter-capacity https://shelteranimalscount.org/
} 


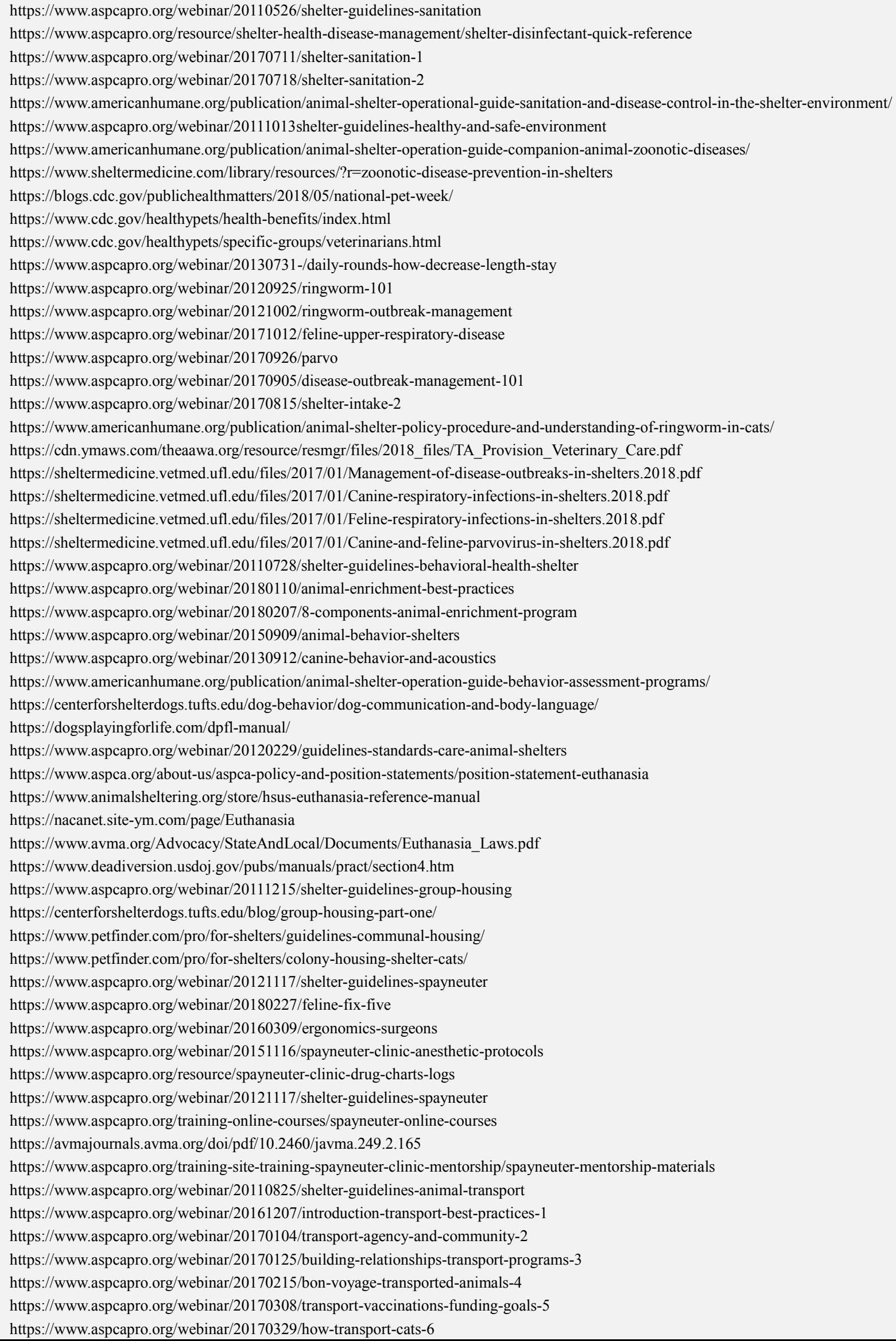




\section{References}

[1] North Carolina Department of Agriculture \& Consumer Services: Public Animal Shelter Report. (2018). Retrieved from

http://www.ncagr.gov/vet/aws/Fix/documents/2018PublicAni malShelterReport3-13-19_000.pdf

[2] Turner P, Berry J, \& MacDonald S. (2012) Animal Shelters and animal welfare: Raising the bar. Canadian Veterinary Journal, 53 (8), 893-896.

[3] Association of Shelter Veterinarians: Guidelines for Standards of Care in Animal Shelters. (2010). Retrieved from https://www.sheltervet.org/assets/docs/shelter-standards-oct20 11-wforward.pdf

(2016).

Retrieved from http://nasphv.org/Documents/NASPHVRabiesCompendium.p df

[8] Bliss, M. \& Dalto, J. (2019) Online training: can it improve safety culture? Professional Safety 64 (1), 54-55.

[9] Brooks, H. L., Pontefract, S. K., Vallance, H. K., Hirsch, C. A., Hughes, E., Ferner, R. E., Marriott, J. F., \& Coleman, J. J. (2016) Perceptions and impact of mandatory eLearning for foundation trainee doctors: a qualitative evaluations. PloS ONE, 11 (12), E0168558.

[10] Wood, S. J., Rogers, M., Frost, M., Revere, D., Rose, B. \& D'Ambrosio, L. (2019) Enhancing access to quality online training to strengthen public health preparedness and response. Journal of Public Health Management and Practice, 25 (6), E1-E9.

[4] Farm Animal Welfare Council: Five Freedoms. (2009). Retrieved from https://webarchive.nationalarchives.gov.uk/20121010012427/ http://www.fawc.org.uk/freedoms.htm

[5] Humane Society of the United States: The HSUS Shelter Advocate Toolkit - All Shelters are not Alike - The Important Differences that can affect the Mission. (2012). Retrieved from https://www.humanesociety.org/sites/default/files/docs/all-shel ters-are-not-alike.pdf

[6] Association of Shelter Veterinarians: Shelter Terminology. (2017). Retrieved from https://www.sheltervet.org/assets/PDFs/shelter\%20terminolog y.pdf

[7] National Association of State Public Health Veterinarians: The Compendium on Animal Rabies Prevention and Control.

[11] U.S. Department of Education. (2010). Retrieved from https://www2.ed.gov/rschstat/eval/tech/evidence-based-practic es/finalreport.pdf

[12] Federman, J. E. (2019) Interruptions in online training and their effects on learning. European Journal of Training and Development. 43 (5), 470-484.

[13] Montgomerie, K., Edwards, M. and Thorn, K. (2016) Factors influencing online learning in an organizational context. Journal of Management Development. 35 (10), 1313-1322.

[14] Pappas, C.: 8 tips to create online training courses for stressed employees. (2016) Retrieved from: https://elearningindustry.com/tips-create-online-training-cours es-stressed-employees

[15] Wulf, J., Blohm, I., Brenner, W. \& Leimeister, J. M. (2014) Massive open online courses. Bus. Inf. Syst. Eng., 6, 111-114. 